\title{
Cuidados paliativos: definição e estratégias utilizadas na prática médica
}

\author{
Palliative care: definition and strategies used in medical practive \\ Cuidados paliativos: definición y estratégias utilizadas en la práctica médica
}

Recebido: 29/12/2020 | Revisado: 30/12/2020 | Aceito: 05/01/2021 | Publicado: 07/01/2021

\author{
Alexandre Ernesto Silva \\ ORCID: https://orcid.org/0000-0001-9988-144X \\ Universidade Federal de São João Del Rei, Brasil \\ E-mail: alexandresilva@ufsj.edu.br \\ Maria Angélica Martins Guimarães \\ ORCID: https://orcid.org/0000-0001-5200-663X \\ Universidade Federal de São João Del Rei, Brasil \\ E-mail: angelica.mg91@yahoo.com.br \\ Rafael Cotta Carvalho \\ ORCID: https://orcid.org/0000-0002-9623-0198 \\ Universidade Federal de São João Del Rei, Brasil \\ E-mail: rcc.med9@gmail.com \\ Thayane Vieira Carvalho \\ ORCID: https://orcid.org/0000-0001-8644-8954 \\ Faculdade Asa de Brumadinho, Brasil \\ E-mail: thayanevieira100@yahoo.com.br \\ Stefãne Amorim Ribeiro \\ ORCID: https://orcid.org/0000-0001-9702-1913 \\ Faculdade Asa de Brumadinho, Brasil \\ E-mail: stefanearibeiro@yahoo.com.br \\ Matheus Rodrigues Martins \\ ORCID: https://orcid.org/0000-0002-3739-6921 \\ Universidade Estadual do Sudoeste da Bahia, Brasil \\ E-mail: matheusrodrigues355@gmail.com
}

\begin{abstract}
Resumo
$\mathrm{O}$ termo cuidado abrange práticas e significados diversos, variando de acordo com a cultura a qual se insere. O cuidado paliativo apresenta-se como uma esfera do cuidado, que surgiu com o objetivo de suprir a uma demanda crescente de pacientes fora das possibilidades de cura terapêutica e que vinham sendo marginalizados e mal assistidos pelo modelo tecnicista da Medicina, com foco na doença em detrimento do doente. O objetivo desse artigo é definir o cuidado paliativo na perspectiva de médicos que atuam em uma unidade oncológica de um hospital de grande porte e as estratégias utilizadas para operacionalizar esta modalidade de assistência à saúde. Trata-se de um estudo qualitativo, de caráter descritivo, realizado com 14 médicos atuantes numa unidade de oncologia de um hospital da região Centro-Oeste de Minas Gerais, seguindo critérios de inclusão e exclusão e, posteriormente, dividido em três categorias de análise: definição dos cuidados paliativos; a fragilidade da formação médica em cuidados paliativos; multidisciplinaridade e a operacionalização do cuidado. Este estudo aponta que o conhecimento em cuidado paliativo ainda é fragilizado e superficial, demonstrando defazagem no ensino médico quando tomamos como referência esse tema. Assim, estratégias de capacitação profissional são necessárias para que esta modalidade de cuidado preste assistência ao indivíduo seguindo o princípio da integralidade. Para que seja eficaz, a operacionalização desse cuidado biopsicossocial e espiritual demanda diálogo por meio do trabalho interdisciplinar, constituindo como ferramenta promissora o Plano Terapêutico Singular (PTS).
\end{abstract}

Palavras-chave: Cuidados paliativos; Educação médica; Medicina paliativa.

\begin{abstract}
The term care encompasses different practices and meanings, varying according to the culture to which it belongs. Palliative care presents itself as a sphere of care, which emerged with the objective of meeting a growing demand from patients outside the possibilities of therapeutic cure and who were being marginalized and poorly assisted by the technical model of Medicine, focusing on the disease in to the detriment of the patient. The purpose of this article is to define palliative care from the perspective of doctors who work in an oncology unit in a large hospital and the strategies used to operationalize this type of health care. This is a qualitative, descriptive study, carried out with 14 doctors working in an oncology unit of a hospital in the Midwest region of Minas Gerais, following inclusion and exclusion criteria, and later divided into three categories of analysis: definition of palliative care; the weakness of medical training in palliative care; multidisciplinarity and the operationalization of care. This study points out that knowledge in palliative care is still weak and superficial, demonstrating a gap in medical teaching when we take this theme as a reference. Thus, professional training strategies are necessary for this type of care to provide assistance to
\end{abstract}


the individual following the principle of integrality. In order to be effective, the operationalization of this biopsychosocial and spiritual care requires dialogue through interdisciplinary work, constituting the Singular Therapeutic Plan (PTS) as a promising tool.

Keywords: Palliative care; Medical education; Palliative medicine.

\section{Resumen}

El término cuidado engloba diferentes prácticas y significados, que varían según la cultura a la que pertenece. Los cuidados paliativos se presentan como un ámbito asistencial, que surgió con el objetivo de atender una demanda creciente de pacientes ajenos a las posibilidades de curación terapéutica y que habían sido marginados y mal atendidos por el modelo técnico de la Medicina, con foco en la enfermedad en en detrimento del paciente. El propósito de este artículo es definir los cuidados paliativos desde la perspectiva de los médicos que trabajan en una unidad de oncología de un gran hospital y las estrategias utilizadas para operacionalizar este tipo de atención de salud. Se trata de un estudio cualitativo, descriptivo, realizado con 14 médicos que laboran en una unidad de oncología de un hospital de la región Medio Oeste de Minas Gerais, siguiendo criterios de inclusión y exclusión, y posteriormente dividido en tres categorías de análisis: definición de cuidados paliativos; la debilidad de la formación médica en cuidados paliativos; multidisciplinariedad y operacionalización de la atención. Este estudio señala que el conocimiento en cuidados paliativos aún es débil y superficial, evidenciando un vacío en la enseñanza médica cuando tomamos este tema como referencia. Así, las estrategias de formación profesional son necesarias para que este tipo de atención brinde asistencia al individuo siguiendo el principio de integralidad. Para ser eficaz, la operacionalización de este cuidado biopsicosocial y espiritual requiere el diálogo a través del trabajo interdisciplinario, constituyendo el Plan Terapéutico Singular (PTS) como una herramienta prometedora.

Palabras clave: Cuidados paliativos; Educación médica; Medicina paliativa.

\section{Introdução}

O termo cuidado, derivado do verbo cuidar, abrange práticas e significados diversos, variando de acordo com a cultura a qual se insere. Etimologicamente, a palavra cuidar é de origem latina, derivada do verbo "cogitare", cujos significados estão relacionados ao pensar, supor e imaginar. Há também aproximação com o vocábulo latino "curare”, cujo sentido associa-se à ideia de "tratar de". O cuidar e o cuidado são próprios do convívio humano, se estabelecem nas diversas esferas da vida em sociedade e são caracterizados por apresentarem uma relação assimétrica, em que não há expectativa de troca (Rabinovich, 2014).

O cuidado em saúde, de acordo com o senso comum, relaciona-se a execução de procedimentos e atitudes orientadas para a manutenção de um corpo livre de doença. No entanto, associam-se os cuidados em saúde como restritos à dimensão do corpo físico, igualado a uma máquina, promove-se um reducionismo que limita, reduz e desencanta esta atividade (Rabinovich, 2014). Assim, o cuidado com o ser humano deve ser amplo e atender aos diferentes aspectos que compõem sua complexidade, ou seja, físico, espiritual, psíquico e social (WHO, 2019).

O cuidado paliativo apresenta-se como uma esfera do cuidado, que surgiu com o objetivo de suprir a uma demanda crescente de pacientes fora das possibilidades de cura terapêutica e que vinham sendo marginalizados e mal assistidos pelo modelo tecnicista da Medicina, com foco na doença em detrimento do doente. O termo cuidado paliativo, cunhado em 1974 pelo canadense Balfour Mount, deriva do latim pallium (“manta”) e remete à ideia de proteger ou amparar(WHO, 2019).

Na prática, os cuidados paliativos correspondem a intervenções orientadas por uma filosofia que abrange o cuidado em seus aspectos físicos, psíquicos, sociais e espirituais, realizadas por equipe multiprofissional. Esta modalidade de cuidado está indicada para pacientes portadores de doença grave e progressiva, que ameace a continuidade da vida e podem ser adequadamente oferecidos a qualquer momento ao longo da trajetória da doença, concomitantes ou não à terapêutica curativa (Firth, O’Brien, Guo, Seymour, Richardson, Bridges, Hocaoglu, et al., 2019).

A Organização Mundial de Saúde (OMS) definiu, em 1990, o termo Cuidado Paliativo e o redefiniu em 2002, explicitando que: "Cuidado Paliativo é uma abordagem que promove a qualidade de vida de pacientes e seus familiares, que enfrentam doenças que ameacem a continuidade da vida, através da prevenção e alívio do sofrimento. Requer a identificação 
precoce, avaliação e tratamento da dor e outros problemas de natureza física, psicossocial e espiritual" (Fernandes, Machado, Sousa, Costa, Queiroz \& Muñoz, 2019).

Todos os pacientes diagnosticados com doenças graves, portadores de diagnósticos suspeitos, prognósticos reservados, àqueles que apresentem necessidade de serem submetidos a procedimentos dolorosos e desconfortáveis, e àqueles que obtiverem fracassos em terapias curativas, são pacientes com indicação para tratamentos paliativos e são expostos diariamente a impactos negativos, juntamente com seus familiares (Silva, Ribeiro, Carvalho, Laia, Ferreira \& Oliveira, 2020).

O envelhecimento progressivo da população contribui para a maior prevalência de doenças crônicas, e estas são as principais causas de morbimortalidade em países como o Brasil, superando as doenças infectocontagiosas. Além disso, o avanço tecnológico alcançado principalmente a partir da segunda metade do século XX, associado ao desenvolvimento da terapêutica, fez com que muitas doenças letais se transformassem em doenças crônicas, levando a longevidade dos portadores dessas doenças, crescendo consequentemente a demanda por cuidados paliativos, exigindo serviços especializados e profissionais treinados e capacitados para assistir adequadamente esta população (Malta, Oliveira, Santos, Andrade \& Silva, 2016; Morin et al., 2017).

Apesar da evidente necessidade de implantação de serviços de cuidados paliativos no sistema de saúde brasileiro, diversos obstáculos se apresentam. Dentre eles, a ausência de uma política governamental que apoie e regularize os cuidados paliativos, a carência de recursos financeiros para pesquisa e desenvolvimento assistencial na área, e deficiência na educação dos profissionais de saúde quanto à temática. Observa-se que no que se refere à formação, educação continuada, treinamento e capacitação, há deficiência de conteúdos para subsidiarem os profissionais de saúde no cuidar de pacientes elegíveis para os cuidados paliativos durante a graduação (Brugugnolli, Gonsaga \& Silva, 2013).

A assistência voltada para os cuidados paliativos inclui medidas que apoiem o paciente psicologicamente, economicamente, socialmente e fisicamente, garantindo o bem estar dos pacientes, de seus familiares e também dos profissionais atuantes nessa área de atendimento (Rodin, Zimmermann, Rodin, al-Awamer, Sullivan\& Chamberlain, 2020).

A educação médica no Brasil enfatiza a formação técnico científica, deixando à margem a abordagem dos aspectos emocionais, sociais e espirituais do indivíduo. Dessa forma, o médico se apresenta, na maior parte das vezes, despreparado para lidar com situações comumente encontradas no cuidado paliativo, como controle de sintomas não físicos da pessoa em fase final de vida, vivência da morte e do luto e apoio aos familiares. Consequentemente, ao se defrontarem com uma situação de terminalidade da vida, estes profissionais sentem-se mais confortáveis ao estabelecer certa distância da pessoa moribunda, de modo a evitar a sensação de frustação e impotência que a morte provoca, prejudicando, assim, o cuidado (Fonseca \& Geovanini, 2013; Goebel, Guo \& Wood, 2016).

A prática desse tipo de cuidado faz emergir importantes reflexões sobre a assistência ao paciente partindo de uma perspectiva para além do curar, mas do cuidar. Diante da fragilidade da formação médica sob este olhar, estratégias de capacitação profissional podem ser utilizadas com a finalidade de melhorar o suporte às pessoas em cuidados paliativos e seus familiares (Fonseca \& Geovanini, 2013; Kissane et al., 2016).

Este estudo teve, portanto, como objetivo conhecer a definição de cuidado paliativo na perspectiva de médicos que atuam em unidade oncológica de um hospital de grande porte e, as estratégias utilizadas para operacionalizar esta modalidade de assistência à saúde.

\section{Metodologia}

Trata-se de um estudo qualitativo, de caráter descritivo. No estudo qualitativo é realizada uma interpretação por parte do pesquisador com suas opiniões sobre o fenômeno em estudo. A coleta de dados na maioria das vezes ocorre por meio de 
entrevistas com questões abertas (Pereira, A. S., Shitsuka, D. M., Parreira, F. J. \& Shitsuka, R, 2018). As pesquisas descritivas têm por objetivo levantar as opiniões, atitudes e crenças de uma população (Gil, 2006).

Foram realizadas entrevistas individuais entre junho a outubro de 2016, com 14 médicos atuantes em uma unidade de oncologia de um hospital de grande porte situado na região ampliada de saúde do Centro-Oeste de Minas Gerais, cujos critérios de inclusão foram: médicos regulamentados pela lei de exercício profissional $\mathrm{n}^{\circ} 12.842$, possuir inscrição no Conselho Regional de Medicina (CRM-MG), ser atuante na unidade de internação citada acima e possuir certificado de residência médica em oncologia. Foram excluídos os médicos oncologistas que estiverem no período de férias ou licença.

A entrevista é uma técnica onde o entrevistador realiza perguntas ao entrevistado como forma de aquisição de informações específicas. Nesse momento se faz a coleta de dados, diagnóstico e orientação (Pereira, A. S., Shitsuka, D. M., Parreira, F. J. \& Shitsuka, R, 2018)

As entrevistas foram gravadas em meios eletrônicos e posteriormente transctitas na íntegra, abordando as seguintes questões norteadoras: Conte o que você conhece sobre Cuidados Paliativos. Qual a fonte deste conhecimento? A partir do conhecimento citado, quais as estratégias você utiliza para operacionalizar os cuidados paliativos?

Dentre os 14 médicos entrevistados, nove eram do sexo masculino e cinco do sexo feminino. A faixa etária destes profissionais variou de 28 a 58 anos, com média de 38,6 anos. Em relação à especialidade médica, cinco eram cirurgiões, destes, dois urologistas, dois coloproctologistas e um cirurgião de cabeça e pescoço, quatro oncologistas, dois radioterapeutas, dois hematologistas e um ortopedista. Quanto ao tempo de atuação em oncologia, houve uma variação de 3 a 23 anos, com uma média em torno de 9,7 anos. Por fim, o tempo de atuação na unidade oncológica do hospital regional variou de 02 semanas a 15 anos, com uma média de 5,6 anos, aproximadamente.

A interpretação dos dados foi realizada a partir da análise de conteúdo, método que se complementa ao estudo das motivações, atitudes, valores, crenças e tendências, ou seja, ao conjunto de técnicas de análise de comunicações desenvolvido por procedimentos sistemáticos e objetivos. Assim, a descrição do conteúdo tem por objetivos apresentar indicadores que permitam a inferência de conhecimentos relativos às condições de produção e/ou recepção das mensagens (Costa, Poles \& Silva, 2016).

Esse método se dá por meio da leitura e interpretação do conteúdo das entrevistas, permitindo a realização de análises que conduzem as descrições objetivas, sistemáticas e qualitativas das comunicações, auxiliando reinterpretações desse conteúdo e atingindo uma compreensão aprofundada de seus significados. Constitui-se em mais que uma simples técnica de análise de dados, já que não busca explicar somente as características e opiniões dos entrevistados, mas o entendimento do sentido e significados atribuídos à problemática focalizada (Costa, Poles \& Silva, 2016).

De acordo com as diretrizes e normas da Resolução CNS/MS466/2012, que regulamentam a ética da pesquisa envolvendo seres humanos no Brasil, a cada um dos sujeitos da pesquisa foi demandado o termo de consentimento livre e esclarecido (TCLE) para participar no estudo, com a assinatura de um termo de consentimento.

Inicialmente, os entrevistados foram informados sobre o conteúdo da pesquisa, seus objetivos, os procedimentos e a possibilidade de recusa. O sigilo das informações bem como a possibilidade dos participantes deixarem o estudo a qualquer momento, mesmo após ter assinado o termo de consentimento sem prejuízos morais ou penalizações lhes foram garantidos, pois os resultados são apresentados sem possibilidade de identificação nominal. Os entrevistados foram numerados sequencialmente em (E1, E2... E12).

O projeto de pesquisa, assim como o TCLE, foi submetido e aprovado pelo Comitê de Ética em Pesquisa da Universidade Federal de São João Del Rei, sob número CAAE 51214415.1.0000.5545. 


\section{Resultados e Discussão}

A organização do material de pesquisa e análise inicial fez emergir três unidades de registro: "formação médica", "multidisciplinaridade" e "atuação profissional". A partir das singularidades inerentes à atuação profissional dos médicos envolvidos no estudo, e da semântica dos discursos, foram construídas três categorias de análise: "Definição dos cuidados paliativos"; "A fragilidade da formação médica em cuidados paliativos"; "Multidisciplinaridade e a operacionalização do cuidado".

\section{Definição dos cuidados paliativos}

A terminologia adotada para descrever o cuidado dispensado a pacientes portadores de doença progressiva mudou ao longo do tempo. Essas adaptações refletem o desenvolvimento histórico do cuidado de final da vida e a mudança dos significados que o conceito sofreu à medida que os conhecimentos nessa área se desenvolveram. As diversas significações que o cuidado de fim de vida assume para profissionais especialistas de diferentes culturas apresentam cunho subjetivo e definições provisórias que enfatizam diferentes elementos. Não existe, portanto, uma definição uniforme na prática e tal fato geram ambiguidades no delineamento do cuidado, como pacientes elegíveis e momento de início (Gysels, Evans, Meñaca, Harding \& Pool, 2013).

O termo "cuidado paliativo" recebe várias definições em diferentes textos fonte, como artigos, dicionários médicos e livros didáticos, porém, não se observa grande discordância entre os conceitos. Existe uma homogeneidade em tal conceituação e a definição mais utilizada é a oferecida pela OMS. Segundo a OMS, o tratamento paliativo é aquele direcionado a pacientes que possuem uma doença incurável e que devem ser acompanhados por uma equipe multidisciplinar (Carvalho et al., 2020; WHO, 2019).

Observa-se uma concepção relativamente uniforme sobre o significado de cuidados paliativos na fala de médicos quando entrevistados. Os discursos trazem, de uma forma geral, a importância da abordagem integral do indivíduo, aproximando-se da definição adotada pela OMS. No entanto, o conteúdo de algumas falas apresenta-se tangencial e pouco consolidado, contribuindo para o pressuposto de que existe, por parte dos entrevistados, fragilidade na conceituação do cuidado paliativo, que apesar de ser considerada subjetiva, está bem sedimentado na literatura médica (Gysels, Evans, Meñaca, Harding $\&$ Pool, 2013).

Evidencia-se inconsistência e confusão por parte dos médicos na definição de "paciente em cuidado paliativo", e que isto se deve à própria imprecisão na definição do termo "paliativo". Tal inconsistência reflete diretamente na identificação e eleição do paciente candidato a esta modalidade de cuidado. Assim, discursos tangenciais e superficiais sobre o conceito de cuidados paliativos podem evidenciar, como consequência, um cuidado ineficiente e fragmentado, pautado em definições que mais se assemelham a reprodução automática de conceitos (Mitchell, Noble, Finlay\& Nelson, 2015).

O paciente destinado aos cuidados paliativos vivencia a experiência de ser acometido por uma doença crônica, progressiva, degenerativa e sem possibilidades atuais de cura, o que desencadeia reflexões e sofrimentos singulares a respeito da finitude da vida, provocando repercussões físicas, psicológicas, sociais e espirituais. Diante das circunstâncias multifatoriais que envolvem estes pacientes, um único provedor de cuidado se faz insuficiente para atender as suas diferentes demandas (Blinderman \& Billings, 2015; Dantas \& Amazonas, 2016).

Desta forma, quando se racionaliza os cuidados paliativos, defende-se a priori um trabalho em equipe, que deve ser formada por profissionais de diferentes especialidades, permitindo assim complementação de saberes, partilha de responsabilidades e a resolução das diversas demandas a partir das especialidades. Existem múltiplos termos aplicados a estas 
equipes de trabalho, dependendo das características de funcionamento destas equipes, sendo os mais abordados a equipe multidisciplinar e a interdisciplinar (Noreika \& Coyne, 2015).

Os entrevistados definiram os cuidados paliativos como suporte assistencial aos aspectos físicos, psicossociais e espirituais, que oferecidos ao paciente com doença grave avançada e sem cura, tem por objetivo proporcionar bem-estar e melhor qualidade de vida ao mesmo.

"Cuidado paliativo é a ferramenta que a gente tem pra trazer conforto pro paciente, né, trazer o bem-estar social, o bem-estar espiritual, analgesia, controle da dor. É transformar aquele momento que ele tem, independente do tempo, que ele possa sobreviver da melhor forma possivel, o mais digno possível."(E2).

“[...] referem-se a todos os níveis de atenção humanitária naqueles pacientes que não tem um recurso terapêutico curativo, não só tratamento medicamentoso." (E5).

Outro aspecto que deve ser destacado no que tange o significado de cuidados paliativos é a incorporação da família como pilar do cuidado. O maior desafio da prática dos cuidados paliativos é a prestação eficaz de cuidados centrados na família. Não se pode ignorar que a família enfrenta múltiplas adversidades diante do diagnóstico de doença que impõe risco à vida e, portanto, não incorporar a família nos cuidados paliativos é realizar assistência, no mínimo, incompleta (Kirby, Kenny, Broom, MacArtney \& Good, 2017; Kim, Carver, Spiegel, Mitchell \& Cannady, 2017). Neste estudo apenas dois dos entrevistados fizeram menção ao papel da família como parte da definição de cuidados paliativos.

"[...] é o cuidado que você institui em um doente que tem uma doença grave e que vai levá-lo a morte e que deve ser implantado dentro de uma unidade oncológica, na verdade desde sua entrada até a saída de sua família, porque cuidado paliativo não termina com a morte do doente, ele deve se estender com os cuidados da família." (E6).

"[...] dar qualidade de vida ao paciente e também à sua família, não se esquecendo dos familiares que cercam esse paciente." (E11).

Um entrevistado mencionou o momento de iniciar os cuidados paliativos.

"[...] cuidado integral ao paciente e, sobretudo ao paciente com câncer ou com qualquer outra doença avançada, não necessariamente na fase terminal, muito pelo contrário, a gente deveria iniciar os cuidados paliativos muito mais precocemente do que a gente faz." (E11).

\section{A fragilidade da formação médica em cuidados paliativos}

Discrepâncias e superficialidade de conceitos podem ser justificadas por uma formação médica acadêmica que pouco aborda o ensino dos cuidados paliativos. O que contribui para a demanda por cuidados paliativos exceder o número de médicos que estão treinados para pôr em prática o cuidado (DiBiasio, 2016; Cripe et al., 2017).

Neste estudo, muitos discursos mencionam como fonte de conhecimento a prática clínica, demonstrando ausência de qualquer preparação ou capacitação prévia em cuidados paliativos. Apenas a prática não é suficiente para consolidação do conhecimento e execução eficaz do cuidado, o que leva a má qualidade da assistência fornecida por médicos clínicos sem formação especializada em cuidados paliativos. Na busca de solucionar o déficit gerado durante a graduação de médicos, métodos de capacitação em cuidados paliativos estão sendo empregados em escolas médicas. Associado ao aperfeiçoamento na 
formação acadêmica observa-se a incrementação de programas de educação continuada dentre os profissionais que lidam com tais cuidados, contribuindo para o aprimoramento das práticas multidisciplinares (Hauser, Preodor, Roman, Jarvis \& Emanuel, 2015; Smith \& Schaefer, 2014).

Os profissionais entrevistados relataram a ausência de preparo em suas graduações para lidar com o paciente em cuidados paliativos e a complexidade que envolve esse tratamento. Diante desse relato, observou-se o reconhecimento da importância do trabalho em equipe multidisciplinar e multiprofissional para operacionalização dos cuidados paliativos.

Quando questionados sobre a origem de seu conhecimento em cuidados paliativos, os discursos dos entrevistados foram variados, evidenciando múltiplas fontes, desde teóricas às baseadas na prática cotidiana.

"São várias fontes né, tem algumas pessoas, alguns autores inspiradores, Kluber-Ross é a mais famosa [...]" (E12).

"Estudo próprio, leitura e interesse pela área [...]mas nada muito formal no sentido de graduação, curso, pósgraduação." (E6).

"[...] não tenho nenhum preparo enfático do ponto de vista de cuidados paliativos, o que tenho é minha experiencia prática trazida desde a faculdade, passando pela residência e agora nos tempos de hoje.” (E1).

"[...] durante a faculdade até o meu contato com oncologia foi bem restrito, na universidade a gente tem as áreas específicas de conhecimento e dentro de cada área, por exemplo, dentro da gastroenterologia a gente vê um pouquinho de câncer, mas praticamente nada mesmo de cuidados paliativos." (E7).

“[...] na verdade a gente sabe que não é um assunto muito difundido, nem na graduação né, é citado, mas não tem uma matéria específica sobre isso nem na própria oncologia." (E10).

Um entrevistado destacou a capacitação de profissionais como possível alternativa para suprir a incipiente abordagem doscuidados paliativos no processo de formação médica.

"Até acho que seria importante o desenvolvimento de programas de capacitação pra esses profissionais que trabalham na área, com palestras né, informação teórica mesmo dentro dos cuidados paliativos."(E1).

\section{Multidisciplinaridade e a operacionalização do cuidado}

Conceitualmente, uma equipe multidisciplinar caracteriza-se pela atuação de diversas disciplinas em torno de um mesmo tema ou problema, sem o estabelecimento de relações entre os profissionais da equipe. As diferentes disciplinas atuam independentes, mas carecem da abordagem das outras e o prontuário é o principal meio de comunicação. Em contraste, nas equipes interdisciplinares duas ou mais disciplinas intencionalmente relacionam-se entre si para alcançar maior abrangência de conhecimento. Nesta organização, existe troca profissional por meio de reuniões regulares para discutir o estado do paciente e evoluir o plano de cuidados. Dessa forma, entende-se que, enquanto na multidisciplinaridade há soma de abordagens, na interdisciplinaridade existe síntese (Porto, Thofehrn, Amestoy, Gonzáles \& Oliveira, 2012).

A abordagem interdisciplinar tem sido estabelecida como a melhor prática para a operacionalização dos cuidados paliativos. Uma intervenção desse tipo de tratamento, com concomitante abordagem interdisciplinar resulta em melhorias estatisticamente significativas na qualidade de vida, nos sintomas e no sofrimento psicológico em pacientes com câncer de 
pulmão, reafirma-se assim a importância da interdisciplinaridade em cuidados paliativos (Ferrell, Sun, Hurria, Cristea, Raz, Kim, Reckampet al., 2015; Porto, Thofehrn, Amestoy, Gonzáles \& Oliveira, 2012).

$\mathrm{Na}$ conceituação de cuidados paliativos, muitos entrevistados deram ênfase à natureza multidisciplinar do cuidado, atribuindo as estas esferas, papel fundamental na operacionalização e execução do conhecimento teórico.

“[...] a operacionalização é pessoal, ela deve ser multidisciplinar, que envolva todos (...) dando este suporte, como te falei fisico, social, psicológico, espiritual, multiprofissional para ele." (E12).

"[...]dar suporte ao paciente, é o suporte médico, o suporte de enfermagem, o suporte multidisciplinar para que este paciente tenha uma qualidade de vida." (E9).

Foi mencionada ainda a dificuldade de operacionalizar os cuidados paliativos em algumas instituições, destacando como causa principal a ausência de um serviço formal que institua o cuidado paliativo.

"Operacionalização de cuidados paliativos no nosso meio é uma operacionalização muito difícil e muito pouco feita. Na prática o que a gente tem oportunidade de fazer? Como não existe assim um cuidado paliativo instituído formalmente acaba-se encaminhando." (E6).

A instituição do trabalho interdisciplinar em cuidados paliativos é uma tarefa complexa, que exige consenso e clareza quanto aos objetivos e estratégias propostas, competência de cada membro da equipe em sua área de atuação, além do estabelecimento de um processo de comunicação eficiente, baseado no diálogo. A comunicação representa um importante instrumento para as práticas interdisciplinares e a sua ineficiência implica em dificuldade no delineamento de responsabilidades e tarefas entre os membros da equipe, falta de integração do conhecimento e desarticulação de ações, comprometendo assim a prática dos cuidados paliativos (Porto, Thofehrn, Amestoy, Gonzáles \& Oliveira, 2012).

Uma estratégia utilizada para operacionalizar os cuidados paliativos e que favorece o trabalho interdisciplinar é a utilização do Plano Terapêutico Singular (PTS). O PTS se apresenta como ferramenta "transformadora" do cuidado e consiste num conjunto de propostas terapêuticas que são articuladas para um indivíduo ou coletividade, objetivando o cuidado integral, elaborado por equipe interdisciplinar. A interdisciplinaridade deve ser reforçada por meio de reuniões regulares entre os membros e pela tomada de decisões em conjunto (Silva, Sousa, Melo, Gouveia, Andrade, Cabral, Pachecoet al., 2013).

\section{Considerações Finais}

Este estudo aponta que o conhecimento em cuidado paliativo ainda é fragilizado e superficial, demonstrando defazagem no ensino médico quando tomamos como referência este tema. Assim, estratégias de capacitação profissional se mostram necessárias para que esta modalidade de cuidado preste assistência ao indivíduo de acordo com o princípio da integralidade. Para que seja eficaz, a operacionalização deste cuidado biopsicossocial e espiritual demanda diálogo por meio do trabalho interdisciplinar, constituindo-se como ferramenta promissora para o mesmo, o Plano Terapêutico Singular.

Como trabalhos futuros, espera-se o desenvolvimento de estudos que direcionem programas com abordagens sobre o tema nos cursos de graduação, pós-graduação e dentro das instituições como Educação Permanente, para garantir que os profissionais melhores qualificados nos atendimentos desses pacientes e na prescrição desse tipo de tratamento. 


\section{Referências}

Blinderman, C. D., \& Billings, J. A. (2015). Comfort care for patients dying in the hospital. The New England Journal of Medicine, 373(26), 2549-2561

Brugugnolli, I. D., Gonsaga, R. A. T., \& Silva, E. M. (2013). Ética e cuidados paliativos: o que os médicos sabem sobre o assunto? Revista Bioética, 21(3), 477-85.

Carvalho, T. V., Nakashima, S. S., Correia, T. L. B. V., Dias, S. B., Silva, M. A., Santos, R. C.\& Pena, H. P. (2020). Eficácia da quimioterapia nos cuidados paliativos em pacientes oncológicos: uma revisão integrativa. Research, Society and Development, 9(11).

Costa, A. P., Poles, K. \& Silva, A. E. (2016). Formação em cuidados paliativos: experiência de alunos de medicina e enfermagem. Interface (Botucatu), 20(59), 1041-1052.

Cripe, L. D., Hedrick, D. G., Rand, K. L., Burns, D., Banno, D., Cottingham, A., Litzelman, D. et al. (2017). Medical students' professionalism narratives reveal that experiences with death, dying, or palliative care are more positive than other experiences during their internal medicine clerkship. The American Journal Of Hospice \& Palliative Care, 34(1), 79-84.

Dantas, M. M. F., \& Amazonas, M. C. L. A. (2016). A experiência do adoecer: os cuidados paliativos diante da impossibilidade da cura. Revista da Escola de Enfermagem da USP, 50(n.esp), 047-053

DiBiasio, E. (2016). Palliative and End-of-Life Care Education Among Alpert Medical School Students. RhodeIsland Medical Journal, 99(4), 20-25.

Fernandes, M. P., Machado, D. B. O. M., Sousa, E. S. S., Costa, T. L., Queiroz, R. B., \&Muñoz, R. L. S. (2019). Autoavaliação do Conhecimento em Cuidados Paliativos por Médicos Residentes de um Hospital Universitário. Rev Fun Care Online, 716-722.

Ferrell, B., Sun, V., Hurria, A., Cristea, M., Raz, D. J., Kim, J. Y., Reckamp, K., et al. (2015).Interdisciplinary palliative care for patients with lung cancer. Journal of Pain and Symptom Management, 50(6), 758-767.

Firth, A. M., O’Brien, S. M., Guo, P., Seymour, J., Richardson, H., Bridges, C., Hocaoglu, M. B. et al. (2019). Establishing key criteria to define and compare models of specialist palliative care: A mixed-methods study using qualitative interviews and Delphi survey.Palliative Medicine, 33(8), 1114-1124.

Fonseca, A., \& Geovanini, F. (2013). Cuidados paliativos na formação do profissional da área de saúde. Revista Brasileira de Educação Médica, 37(1), 120125.

GIL, A. C. (2006). Métodos e técnicas de pesquisa social. (6a ed.), Atlas.

Goebel, J., Guo, W., \& Wood, K. A. (2016). Teamwork and Perceptions of Palliative Care Quality. Journal of Hospice and Palliative Nursing, $18(3)$, $242-248$.

Gysels, M., Evans, N., Meñaca, A., Higginson, I. J., Harding, R., \& Pool, R. (2013). Diversity in defining end of life care: an obstacle or the way forward? Plos one, 8(7), 01-07.

Hauser, J. M., Preodor, M., Roman, E., Jarvis, D. M. \& Emanuel, L. (2015).The Evolution and Dissemination of the Education in Palliative and End-of-Life Care Program. Journal of Palliative Medicine, 18(9), 765-770.

Kim, Y., Carver, C. S., Spiegel, D., Mitchell, H. R. \& Cannady, R. S. (2017).Role of family caregivers' self-perceived preparedness for the death of the cancer patient in long-term adjustment to bereavement. Psychooncology, 26(4), 484-492.

Kirby, E., Kenny, K., Broom, A., MacArtney, J., \& Good, P. (2017). The meaning and experience of bereavement support: A qualitative interview study of bereaved family caregivers. PalliatSupport Care, 16(4), 396-405.

Kissane, D. W., Zaider, T. I., Li, Y., Hichenberg, S., Schuler, T., Lederberg, M., Lavelle, L., et al. Randomized controlled trial of family therapy in advanced cancer continued into bereavement. Journal of ClinicalOncology, 34(16), 1921-1927.

Malta, D. C., Oliveira, T. P., Santos, M. A. S., Andrade, S. S. C. A. \& Silva, M. M. A. (2016). Avanços do Plano de Ações Estratégicas para o Enfrentamento das Doenças Crônicas não Transmissíveis no Brasil, 2011-2015. Epidemiologia dos Serviços de Saúde, 25(2), 373-390.

Mitchell, H., Noble, S., Finlay, I., \& Nelson, A. (2015).Defining the palliative care patient: its challenges and implications for service delivery. BMJ Supportive \& Palliative Care, 3(1), 46-52.

Morin, L., Aubry, R., Fovra, L., MacLeod, R., Wilson, D. M., Loucka, M., Csikos, A. et al. (2017).Estimating the need for palliative care at the population level: A cross-national study in 12 countries. Palliative Medicine, 31(6), 526-536.

Noreika, D. M., \& Coyne, P. J. (2015). Implementing palliative care interdisciplinary teams: Consultative versus integrative models. Critical Care Nursing Clinics of North America, 27(3), 297-306.

Pereira, A. S., Shitsuka, D. M., Parreira, F. J., \& Shitsuka, R. (2018). Metodologia da pesquisa científica.

Porto, A. R.,Thofehrn, M. B., Amestoy, S. C., Gonzáles, R. I. C. \& Oliveira, N. A. (2012). The essence of interdisciplinary practice in palliative care delivery to cancer patients. Investigación y EducaciónenEnfermería, 30(2), 231-239.

Rabinovich, E. P. (2014). Sobre o cuidar e sua rede. DiálogosPossíveis, 11(2), 1-6.

Rodin, G., Zimmermann, C., Rodin, D., al-Awamer, A., Sullivan, R. \& Chamberlain, C. (2020).COVID-19, palliative care and public health.European Journal of Cancer, 136, 95-98. 
Research, Society and Development, v. 10, n. 1, e18810111585, 2021 (CC BY 4.0) | ISSN 2525-3409 | DOI: http://dx.doi.org/10.33448/rsd-v10i1.11585

Silva, A. E., Ribeiro, S. A., Carvalho, T. V., Laia, D. H. S., Ferreira, G. J. \& Oliveira, L. A. (2020). A percepção do profissional enfermeiro frente à comunicação de notícias difíceis. Research, Societyand Development, 9(12).

Silva, E. P., Sousa, M. M., Melo, F. A. B. P., Gouveia, R. A., Andrade, A. F. R., Cabral, A. F. F., Pacheco, M. C. S. et al. (2013). Projeto Terapêutico Singular como estratégia de prática da multiprofissionalidade nas ações de saúde. Revista Brasileira de Ciências da Saúde, 17(2), 197-202.

Smith, G. M., \& Schaefer, K. G. (2014).Missed opportunities to train medical students in generalist palliative care during core clerkships. Journal of Palliative Medicine, 17(12), 1344-1347.

World Health Organization (WHO). (2019). WHO definition of palliative care. Geneva: WHO. 